\title{
Mesure de la vulnérabilité des milieux urbains au Cameroun face au changement climatique
}

C. C. Fandjio Yonzou ${ }^{\mathrm{a}, 1}$, U. J. M. Pettang Nana ${ }^{\mathrm{a}}$, M. B. Manjia ${ }^{\mathrm{a}}$, C. Pettang ${ }^{\mathrm{a}}$

${ }^{a}$ Department of Civil Engineering, National Advanced School of Engineering, University of Yaoundé I. PO Box. 8390, Yaoundé, Cameroun.

Corresponding author: Tel.(+237) 691219062

E-mail address: cabralfandjio@outlook.fr

\section{Résumé}

Les différentes catastrophes survenues en milieu urbain au Cameroun récemment, mettent en exergue les désormais dangers permanents qui pèsent sur les habitants. On note une augmentation des extrêmes : de hautes températures, des perturbations des régimes pluvieux et la récurrence des vents violents. Certains de ces effets sont amplifiés par la géographie contraignante de plusieurs villes et par l'influence anthropique sur l'environnement. En effet, d'une part les sites de plusieurs villes camerounaises ont soit des reliefs accidentés qui favorisent l'érosion des terrains, soit des configurations planes qui limitent l'évacuation gravitaire de l'eau de ruissellement. D'autre part, la croissance urbaine rapide et la prolifération des bidonvilles qui couvrent près de 65 à 70\% de la superficie urbaine, usent plus rapidement les ouvrages et infrastructures d'assainissement urbaines lorsqu'ils existent dus à l'imperméabilisation accélérée et continue du sol urbain ainsi qu'à la mauvaise gestion des déchets. L'urgence de trouver des solutions durables à ce phénomène est désormais sans équivoque. Elle nécessite un encadrement normatif inclusif plus performant que celui actuellement en vigueur, qui amène le pays vers la résilience.

\begin{abstract}
The disasters that've occurred recently in urban areas in Cameroon, highlight the permanent dangers weighing on the inhabitants. There is an increase in extremes climate parameters: high temperatures, rainfall disturbances and the recurrence of gales. Some of these effects are amplified by both the constraining relief of several cities and human influence on the environment. In fact, on the one hand, the sites of several Cameroonian cities either have rugged reliefs that promote land erosion, or flat configurations that limit the gravity discharge of runoff water. On the other hand, the rapid urban growth and the proliferation of slums which cover nearly 65 to $70 \%$ of the urban area, wear out more quickly the works and urban sanitation infrastructures when they exist due to the accelerated and continuous waterproofing soil in addition to the poor waste management. The urgency to find lasting solutions to this phenomenon is now unequivocal. It requires an inclusive normative framework that is more effective than that currently in force, which brings the country towards resilience.
\end{abstract}

Received: 01/07/2021

Accepted: 15/09/2021

DOI: https://dx.doi.org/10.4314/jcas.v17i2.3

(C) The Authors. This work is published under the Creative Commons Attribution 4.0 International Licence. 


\section{Introduction}

Le monde connait des variations extrêmes du climat dues en grande partie à l'enrichissement de l'atmosphère en gaz à effet de serre (GES) dont le plus prépondérant est le gaz carbonique venu des villes (H. Waisman, 2020 ; A. Sharifi, 2021). Ce dérèglement explique les sécheresses, les tempêtes, les inondations et incendies dévastateurs observés dans tous les coins du globe terrestre (V. LEFEBVE, 2020 ; B. Swynghedauw et J.-L. Weméau, 2021). Ces variations ont des impacts significatifs sur les secteurs économiques, les ressources naturelles, les écosystèmes, les revenus, et la santé (GIEC, 2014a ; (B. Swynghedauw et J.-L. Weméau, 2021). Cette situation met en exergue la vulnérabilité des milieux urbains face au changement climatique, intensifiée ou non par le niveau de développement des pays (GIEC, 2012 ; M. Lawhon, 2014 ; C. Brand et al., 2021). Dans les villes des pays en voie de développement (PED) notamment du Cameroun, son ampleur est de plus en plus surprenante et se traduit par des manifestations néfastes du changement climatique accentuées par une urbanisation incontrôlée (C. Pettang, 2001 ; ONU-HABITAT, 2014). La récurrence et la violence des évènements paroxysmiques à l'instar des inondations couvrant plusieurs centaines de $\mathrm{km}^{2}$, des arrachements de toitures, des renversements des poteaux, support des réseaux techniques et le dessouchement d'arbres par les vents, et des glissements de terrain sont de plus en plus courant chaque année. Bien que d'un point de vue purement économique, les pertes dues à ces conditions météorologiques extrêmes sont mesurées plus importantes dans les pays à revenu élevé (Munich Re, 2004 ; Hallegatte et al., 2013), on note que les incidences les plus graves atteintes et/ou pertes en vies humaines se trouvent dans les PED (UNISDR, 2009 ; FICR, 2010 ; S. Balica et al., 2012). On a relevé que les populations occupant des établissements informels sont généralement plus exposées aux événements climatiques avec une capacité limitée ou inexistante de faire face aux risques (GIEC, 2012).

Le présent article expose et quantifie les manifestations néfastes de plus en plus persistants du changement climatique en milieu urbain en prenant le cas du Cameroun, tout en mettant en exergue l'effet amplificateur des spécificités de l'urbanisation sur ces derniers. L'ampleur quantifiée de ce phénomène ouvre les voies d'atténuation des points faibles du cadre normatif resté jusqu'ici imprécis, lesquels bloquent l'engrenage fluide des propositions de transition vers une urbanisation durable.

\section{Matériels et Méthodes}

\subsection{Description de la zone d'étude}

Situé au cœur du continent africain, le Cameroun s'étend entre les parallèles de latitude Nord $01^{\circ} 40^{\prime}$ et $13^{\circ} 04^{\prime}$; et les méridiens de longitude Est $08^{\circ} 40^{\prime}$ et $16^{\circ} 10^{\prime}$. Dans ce pays, le changement climatique trouve un terrain propice à l'aggravation de ses effets du fait même de son relief tel que l'on peut observer sur la carte du relief du Cameroun de la Figure 1. En effet, on a d'une part des reliefs à fortes pentes constitués d'inselbergs et de montagnes couvrant 63\% du territoire (P. Tchawa, 2012). D'autre part, on a des zones de basse altitude (inférieure ou égale à $800 \mathrm{~m}$ ) et de pente faible (inférieure à $1 \%$ ) en l'occurrence dans les plateaux et les plaines (P. Tchawa, 2012). Quant à la pédologie de la zone d'étude, on remarque l'existence des sols sabloargileux principalement au Nord et au Littoral qui ont un fort taux de ruissellement du fait de leur faible capacité de rétention en eau. Sur le plan humain, on enregistre une forte natalité par rapport à la moyenne mondiale avec un taux de natalité de 38,2\%o habitants au Cameroun contre 19,6\%o sur le plan mondial. 


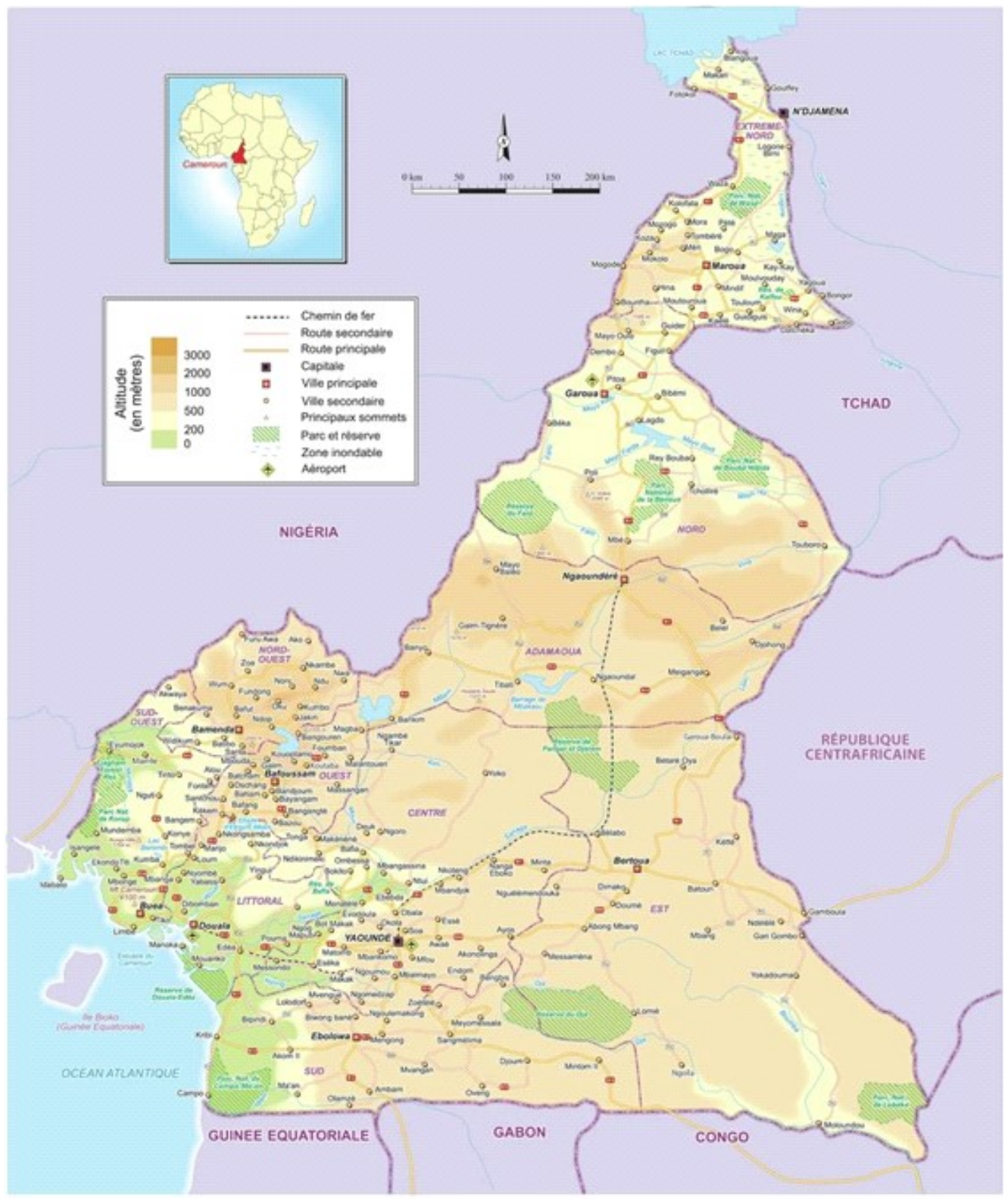

Figure 1: carte du relief du Cameroun (@GeoCameroun)

\subsection{Matériels}

Les outils exploités dans le cadre de cette étude sont les documents de référence issus des travaux déjà publiés. A la base nous avons pris en compte la réglementation existante en matière de gestion des villes dont les Plans Directeurs de Yaoundé et de Douala en font partie. Il s'est aussi agi des relevés des hauteurs de pluie à Yaoundé entre 1981 et 2014 ; des relevés des hauteurs de pluie à Douala entre 1984 et 2018 ainsi que le recueil des inondations à Yaoundé et à Douala entre 1980 et 2019. Également, les plans directeurs de Yaoundé et de Douala ont aussi été utilisés comme source d'informations. Les illustrations photos ont été prises sur internet et sur le terrain par un téléphone portable de marque INFINIX Note4. Afin d'analyser les données et produire les graphiques synthétiques, les logiciels MS EXCEL 2016 et AutoCAD 2014 ont été mis à contribution. 


\subsection{Méthodes de traitement des données}

Les données recensées ont permis de décrire les manifestations du changement climatique et de mesurer sensiblement leur impact sur les infrastructures urbaines et l'habitat. Pour y parvenir nous avons mis en parallèle les paramètres enregistrés sur le climat avec les effets néfastes observés dans quelques villes du Cameroun notamment à Yaoundé dans la région du Centre, Douala dans le Littoral, Maroua et Maga dans l'Extrême-Nord, Bafoussam à l'Ouest, ainsi que Bertoua et Batouri à l'Est. Les données les plus complètes et disponibles étaient celles des deux capitales.

Afin de comprendre les variations des débits maxima aux exutoires des bassins versants urbains, nous avons considéré la formule de Caquot qui est la plus fréquemment utilisée. Elle se définit comme suite :

$Q_{p}(T)=K(M, T)^{\frac{1}{u(T)}} \times I^{\frac{v(T)}{u(T)}} \times C^{\frac{1}{u(T)}} \times A^{\frac{w(T)}{u(T)}}$

(Equation 1)

Où $Q_{p}(T)$ : Débit pour une période de retour T en $\left(\mathrm{m}^{3} / \mathrm{s}\right) ; I$ : Pente moyenne du bassin versant $(\%) ; A$ : Surface du bassin versant (ha) ; et $u(T), v(T), w(T)$, et $K(M, T)$ des paramètres de calage.

La Pression dynamique $q$ (bar) exercée par un vent à la vitesse $v$ s'exprime la manière suivante :

$q=\frac{1}{2} \rho \cdot v^{2}$ où $\rho$ masse volumique de l'air

(Equation 2)

La surface urbanisée à l'année $n$ se déduit de la surface passée au moyen de la relation suivante :

$S_{n}=S_{\text {init }}(\tau+1)^{n}$

(Equation 3)

Avec $\tau$ le taux de croissance en $h a / a n$

Enfin, nous avons analysé suivant le diagramme de (REN Guo-Yu, 2015), l'impact qu'ont actions anthropiques directes et indirectes dans l'accentuation des phénomènes climatiques extrêmes, qui à leur tour détruisent l'environnement de vie et perturbent les habitudes des hommes (Figure 2).

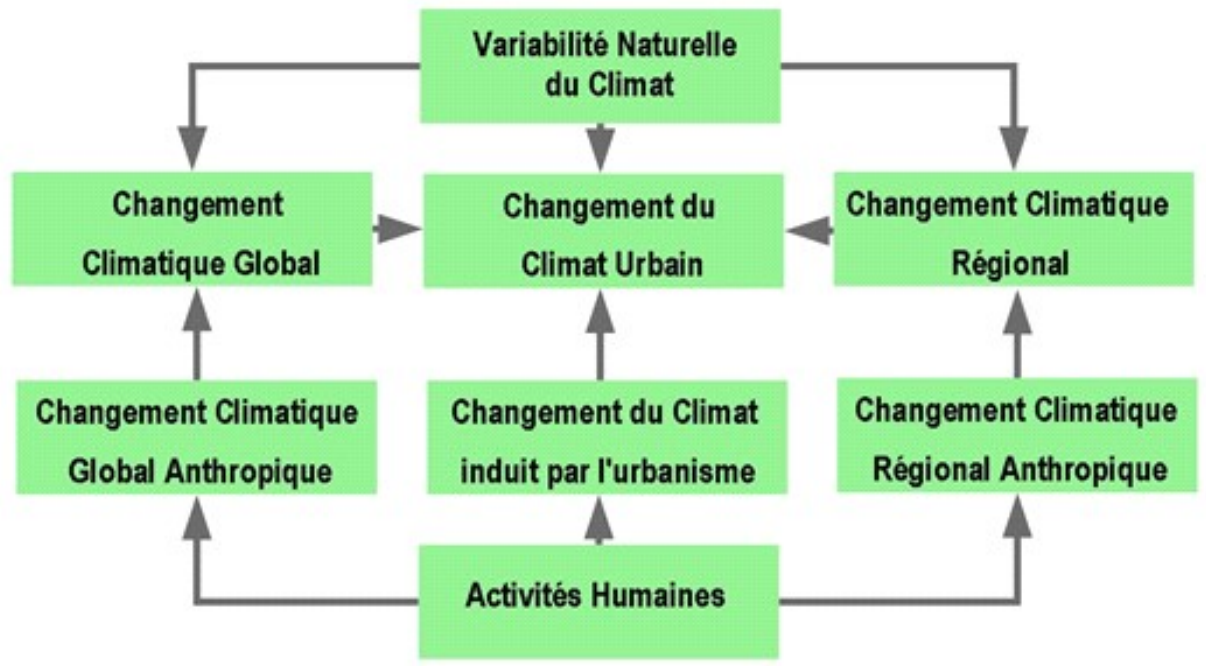

Figure 2: Diagramme schématique des interactions entre des différents niveaux d'influence spatial du changement climatique et leur variabilité (REN Guo-Yu, 2015) 


\section{Résultats}

Il est question de mettre en évidence les différentes manifestations du changement climatique dans les villes; ainsi que la mesure de leur impact sur les infrastructures urbaines et l'habitat au Cameroun.

3.1. L'augmentation de la température et du degré hygrométrique

Le (GIEC, 2014a) note une augmentation de la température mondiale de plus de $4^{\circ} \mathrm{C}$, ce qui se manifeste différemment dans les régions en fonction des zones climatiques. Le constat général dans les différentes villes est qu'il règne désormais des ambiances de plus en plus variées durant des périodes plus ou moins longues. Selon les prévisions faites par le (PNUD, 2008), on serait arrivé en 2020 à une augmentation globale de $0,9^{\circ} \mathrm{C}$ avec un pic en zone soudano-sahélienne allant jusqu'à $2,4^{\circ} \mathrm{C}$.

Plus spécifiquement, la température moyenne annuelle au sol fut de $26,45^{\circ} \mathrm{C}$ en 1984 à Douala et Tiko (G. L'HOSTIS, 1987), cependant en 2000 elle était de $27,75^{\circ} \mathrm{C}$, pour finir entre $29,5^{\circ} \mathrm{C}$ et en 2010 (MINEPDED, 2015). On note une accentuation de l'effet d'ilot de chaleur urbaine comme dans la plupart des centres urbains denses dans le monde (D. Crawley, 2008 ; L. Cui et J. Shi, 2012) en conséquence il fait très chaud, d'où le recours généralisé et récurrent à l'air conditionné, dont l'un des constituants dégrade la couche d'ozone et par ricochet amplifie davantage le réchauffement climatique.

Cette chaleur provoque sur le sol et les chaussées des fissures anormales et précoces, ce qui facilite la désagrégation des couches superficielles de chaussées sous l'effet du trafic et de l'eau. Les voiries et particulièrement celles en terre, deviennent alors des sources de pollution continue avec la poussière soulevée en permanence par les véhicules en circulation. Puisque ces particules fines ne sont plus solidaires de la couche de roulement, les plus gros grains se détachent aisément lors du ruissellement de l'eau, débouchant alors plus rapidement aux pathologies d'arrachement telles que les nids de poule ou les pelades sur les chaussées.

L'humidité de l'air ambiant a tendance à beaucoup varier concomitamment aux variations de températures et aux anomalies de la pluviométrie. Selon le CCHST (Centre Canadien d'Hygiène et de Sécurité au Travail), les irritations des muqueuses du nez et de la gorge, voire des difficultés respiratoires observées chez certaines personnes à priori saines, s'expliquent par un taux d'humidité de l'air trop faible $\left({ }^{\varphi}<20 \%\right)$. Tandis que les élévations anormales du taux d'humidité ( $>70 \%$ ) en saison sèche limitent le refroidissement corporel par transpiration et donc amplifie la sensation de chaleur. La nature de l'air à Douala ou Yaounde au Cameroun par exemple (LGSC, 2019) où on relève un taux minimal de $69 \%$ et de $68,27 \%$ en pleine saison sèche respectivement.

Ces anomalies sur l'air ambiant s'accompagnent aussi de perturbations sur les courants d'air qui balaient les villes.

\subsection{La récurrence des vents violents et leurs conséquences}

Lorsque l'on sait que la pression aérodynamique du vent est proportionnelle au carré de sa vitesse (équation 2), la hausse des vitesses implique des élévations beaucoup plus importantes des actions exercées sur les surfaces des constructions. Les vitesses des vents sont désormais très élevées et plus fréquentes durant les fortes pluies et les tempêtes.

Dans les villes côtières en 1984, on enregistrait à $10 \mathrm{~m}$ d'altitude des vents de moins de $6 \mathrm{~m} / \mathrm{s}$ avec 50\% des cas enregistrés à $2 \mathrm{~m} / \mathrm{s}$ et $95,4 \%$ 
en dessous de $4 \mathrm{~m} / \mathrm{s}$; alors qu'en 2016, la moyenne des vents (50\% des cas) se situe entre $3 \mathrm{~m} / \mathrm{s}$ et $5 \mathrm{~m} / \mathrm{s}$ (J. L. Nsouandélé et al., 2016).

Les conséquences sont l'arrachement des feuilles de tôles et des charpentes des maisons

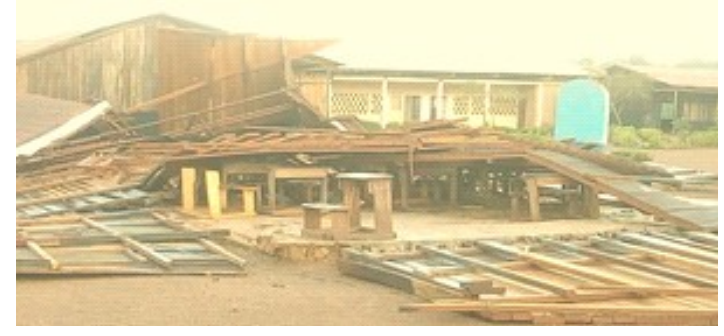

a. Le lycée de Sambo à Batouri sinistré à l'EST du pays, juillet 2019 Cactucameroun.com et immeubles comme l'illustrent les photos de la Figure 3 ci-après.

Figure 3 : Illustrations photos des toitures arrachées par les vents violents

\subsection{Les inondations et leurs conséquences}

\subsubsection{L'effet de l'accroissement du régime pluvieux}

Dans l'ensemble du pays, bien que l'on observe une régression de la pluviométrie de 2,2\% par décennie [PNUD, 2008], la saison pluvieuse est devenue plus courte mais ses épisodes sont plus concentrés et plus forts. L'expérience permet de confirmer que les périodes de retour se réduisent [A. Mailhot et al., 2007]. Cela augmente les occurrences des pluies de forte intensité telles qu'illustrent les répartitions annuelles passées et présentes des pluies sur les graphes de la Figure 4. La récurrence de ces pluies diluviennes remet en cause la capacité à évacuer les eaux de certains ouvrages hydrauliques. En effet, selon les méthodes classiques de calcul de débit (la méthode rationnelle, la méthode de Caquot) exprimée par l'équation 1 de la méthodologie, le débit de dimensionnement des ouvrages hydrauliques est une fonction croissante de l'intensité de pluie, elle-même dépendante de la période de retour choisie. En fait, la tendance générale témoigne une augmentation des débits depuis 2005 de plus de $50 \%$ par an.

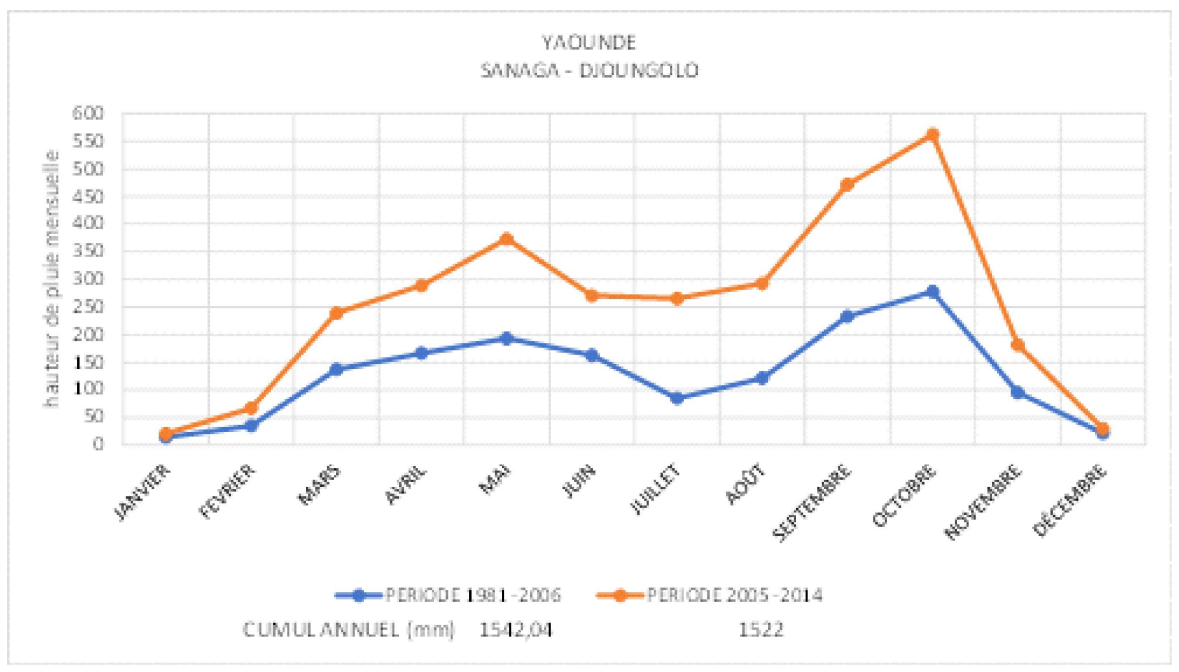

(a) Yaounde entre 1981 et 2014 


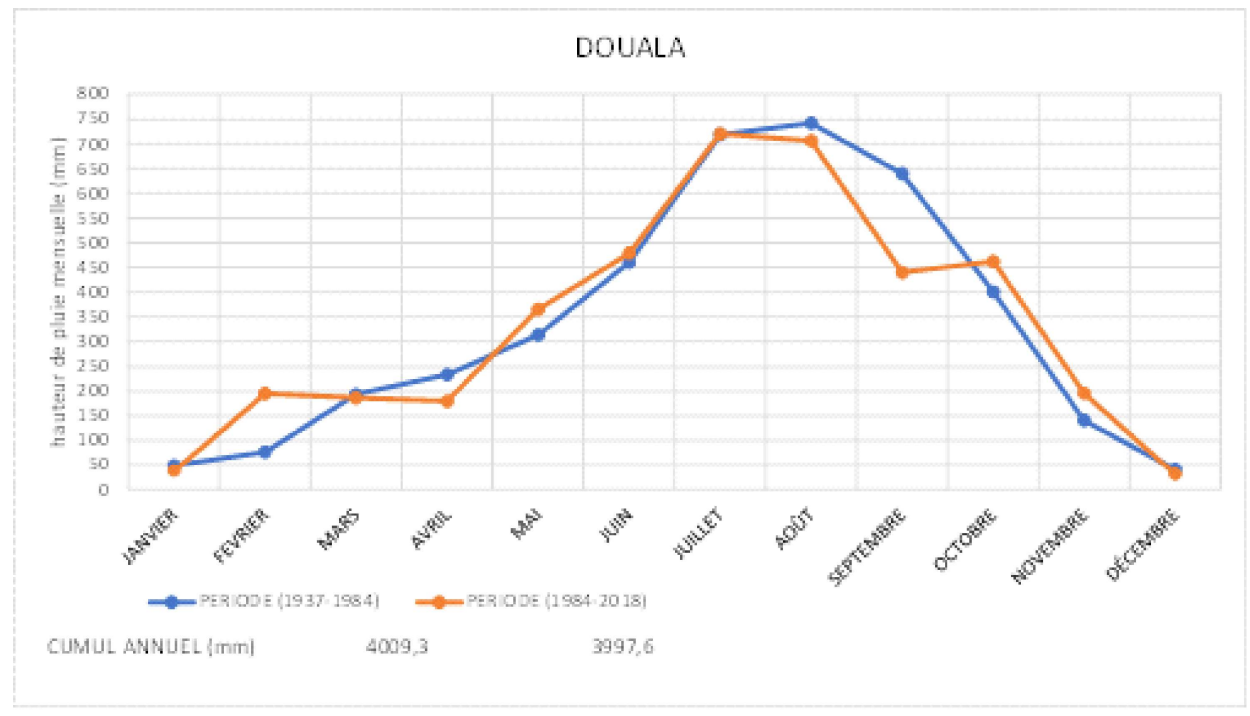

(b) Douala entre 1937 et 2018

Figure 4: Répartition annuelle de la pluviométrie à Douala et Yaoundé

3.3.2. Les effets d'une forte croissance urbaine incontrôléeOn estime par exemple un taux de croissance de 530 ha/an à Yaoundé et de 450 ha/an à Douala dans leur plan directeur respectif, pour la période de 2005 à 2017. A partir de l'équation 3, on calcule l'évolution du périmètre et les résultats sont mis ensemble dans le tableau 1 qui suit.

Tableau 1 : Estimation de croissance de la population et de la surface des aires urbaines de Yaoundé et

Douala

\begin{tabular}{|l|l|l|l|l|l|}
\hline \multicolumn{2}{|l|}{ Population } & $\mathbf{1 9 5 0}$ & $\mathbf{2 0 0 5}$ & $\mathbf{2 0 1 5}$ & $\mathbf{2 0 3 0}$ \\
\hline \multirow{3}{*}{ Douala } & Démographie (hbts) & 95.000 & 1.876 .000 & 2.972 .000 & 5.112 .000 \\
\cline { 2 - 6 } & Superficie (ha) & 1.630 & 16.300 & 20.650 & 24.000 \\
\hline Yaoundé & Démographie (hbts) & 32.000 & 1.781 .000 & 3.097 .000 & 5.734 .000 \\
\cline { 2 - 6 } & Superficie (ha) & 1.740 & 15.900 & 18.320 & 23.620 \\
\hline
\end{tabular}

Source : (populationstat, 2020) ; PDU Yaoundé 2020 ; PDU Douala 2025 ; (LGSC, 2019)

Étant donné que les mouvements de l'eau en surface dépendent de la géologie du milieu et de la typologie du revêtement du sol (S. Andrei, 1977), cette imperméabilisation a pour conséquence directe l'augmentation du taux de ruissellement. Et partant, le coefficient de ruissellement qui est intrinsèque au milieu d'écoulement, devient plus difficile à évaluer. En effet, le coefficient de ruissellement est de l'ordre de 78\% dans le Mfoundi à Yaounde (S. Kuetche, 2013). Les débits résultants s'en voient décuplés. A titre d'illustration on est passé des débits annuels de la Mefou à Nsimalen de $3,8 \mathrm{~m}^{3} / \mathrm{s}$ en 1981 à 17,83 m³/s en 2007 (S. Kuetche, 2013); ou pour le cas du collecteur de Djoungolo à Yaoundé de $13,78 \mathrm{~m}^{3} / \mathrm{s}$ à $31,63 \mathrm{~m}^{3} / \mathrm{s}$ entre 2005 et 2018 (C. Wache, 2018). A côté du relief, la pédologie de la zone d'étude met aussi en exergue des sols sablo-argileux principalement au Nord et au Littoral, qui ont une capacité de rétention de l'eau limitée (peu d'infiltration) à cause de la prédominance de la phase sableuse (O. Leumbe et al., 2015 ; S. Andrei, 1977). 
Cette croissance urbaine est à la fois le résultat d'une forte natalité et aussi de l'exode rural. En effet, la ville actuelle est devenue le refuge des populations rurales fuyant les effets du changement climatique (Banque Mondiale, 2017), puisque les paysans deviennent de plus en plus victimes soit des inondations soit de la sécheresse de leurs champs (GIEC, 2020). Cette situation se confirme par l'indice de pauvreté en zone rurale qui a augmenté de 52,1 à 56,8 en 2014 et le taux d'occupation des villes qui s'est accru, quittant de $37,9 \%$ en 1987 pour $52 \%$ en 2010 selon les chiffres de l'INS en 2015. La situation de la ville est devenue la gestion du paradoxe entre l'accroissement substantiel de la population et la réduction drastique des revenus, situation qui du reste se traduit par une forte dégradation du tissu urbain. Les QHS (quartier à habitats spontanés) tels qu'illustrés sur la Figure 5 prolifèrent alors de manière très significative, leur proportion est partie de 67\% de l'espace urbain en 2007 (ONUHABITAT, 2007) à 75\% en 2019 (LGSC, 2019). Dans ce contexte, les documents de planification urbaine lorsqu'ils existent deviennent difficiles d'opérabilité.

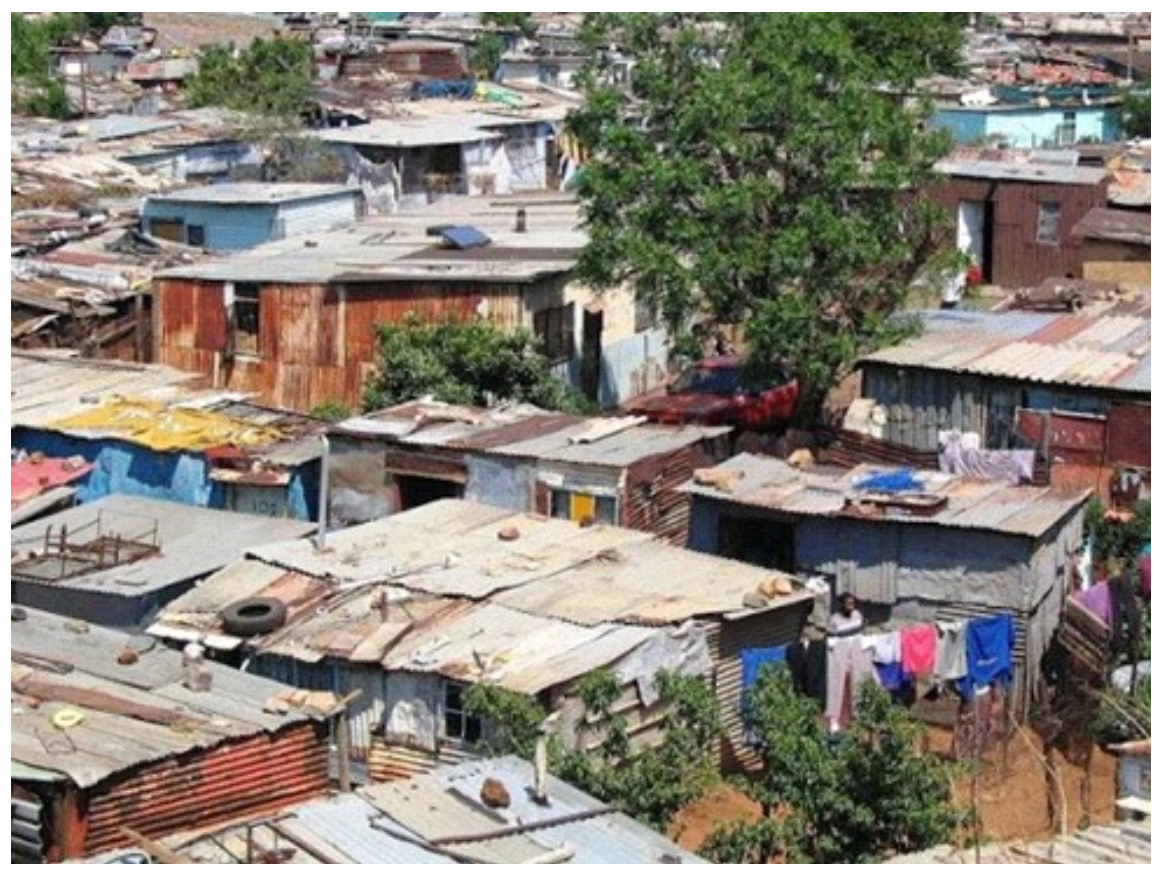

Figure 5 : Bidonville à Etam-Bafia, quartier à Yaoundé [CAjafenews]

La répartition résultante des espaces urbains est par conséquent de plus en plus désordonnée, ce qui rend le coefficient de ruissellement difficile à évaluer car sa valeur est intrinsèque au milieu d'écoulement. C'est pourquoi les coefficients forfaitaires attribués aux tissus urbains tels que recommandés dans certaines normes générales d'hydraulique ne cadrent pas toujours avec le contexte actuel de l'urbanisation.

En outre plus de 66\% de l'espace des QHS est bondé d'ordures (G. TJOMB, 2018). Les populations déversent dans la nature n'importe quel type de déchets ménagers et assimilés principalement de nature plastique (Figure 6), lesquels obstruent les voies d'évacuation des eaux de ruissèlement puisque n'étant pas collectés (E. Ngninkam et E. Tanawa, 2006) et remettent davantage en cause des méthodes traditionnelles de calcul des ouvrages hydrauliques longtemps éprouvés (J. Nyaka, 2017). C’est ce qui explique les cas des inondations survenues bien que les hauteurs interannuelles de pluie étaient faibles, en l'occurrence elles étaient inférieures à $2500 \mathrm{~mm}$ telles que reportées sur la Figure 7 à partir de 2007 (S. Kuetche, 2013). 

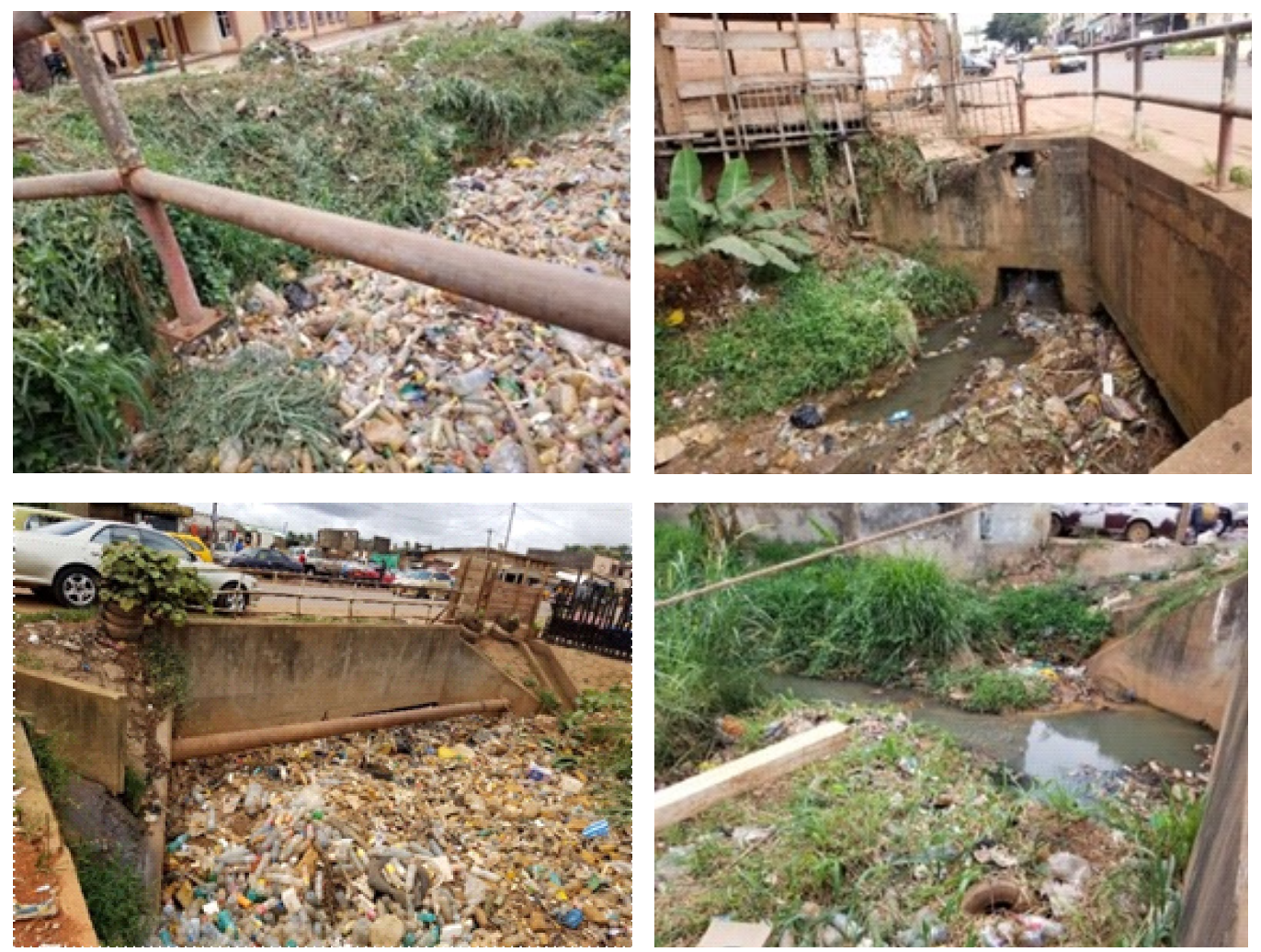

Figure 6 : Illustrations photos de l'obstruction du drain par les déchets solides, à Acacia Yaoundé

Cette description est relativement la même dans la plupart des villes du Cameroun, à la différence que l'intensité du phénomène est proportionnelle à la taille de l'agglomération. La situation résultante est la croissance des inondations dans l'ensemble du pays. L'exemple de l'évolution décennale dans la ville de Douala, qui peut être représentative de la pire situation à l'échelle du pays est mise en exergue par la Figure 7 suivante.

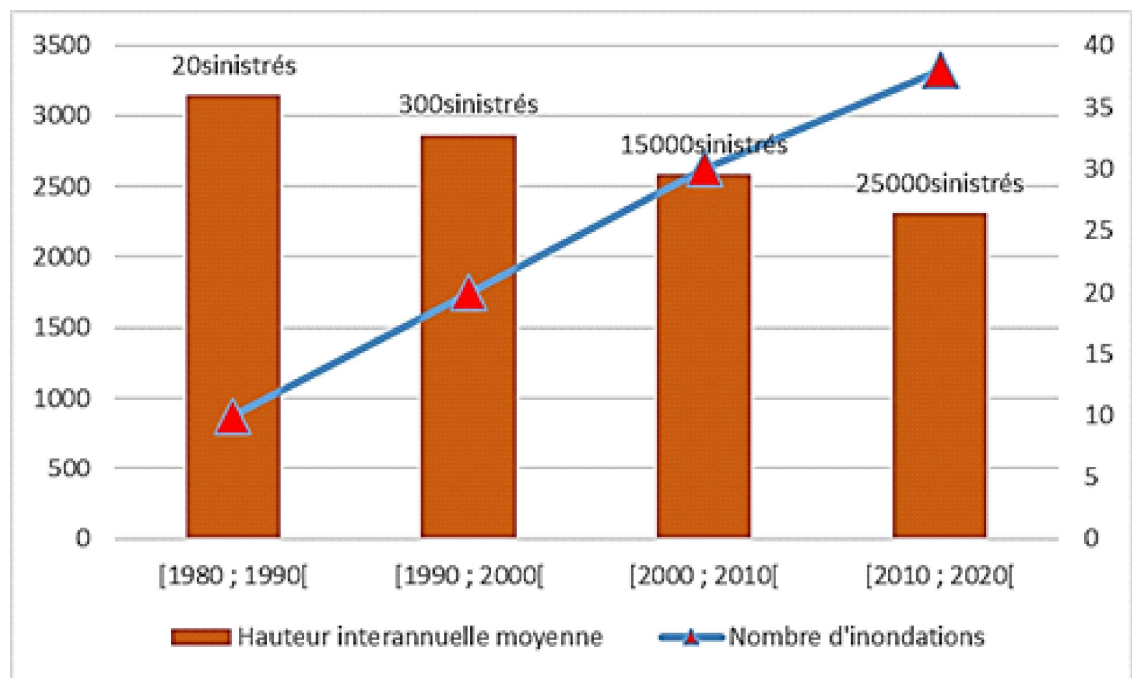

Figure 7: hauteur interannuelle de pluie croisée aux inondations à Douala de 1980 à 2019 


\subsection{Le relief contraignant des sites de plusieurs villes}

Dans les zones de fortes pentes, il surviendra d'avantages des mouvements de terrain lorsque les dispositions constructives ne sont pas respectées (MINEPDED, 2015). Exemple pris sur la Figure 8 de deux cas de glissement de terrain survenus en 2019 à Bafoussam et à Yaoundé.

On a aussi des zones de basse altitude (inférieure ou égale à $800 \mathrm{~m}$ ) et de pente faible (inférieure à $1 \%$ ) qui sont plus vulnérables aux inondations. Les sols sablo-argileux principalement au Nord et au Littoral, qui ont une capacité de rétention de l'eau limitée (peu d'infiltration).

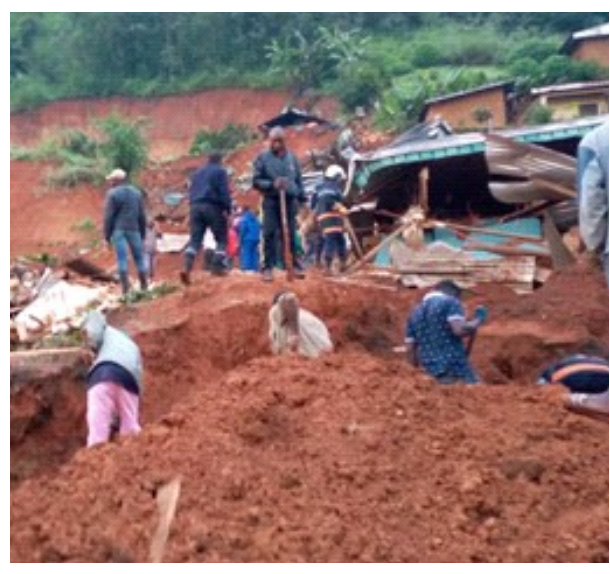

(1) Ngouache à Bafoussam en octobre 2019

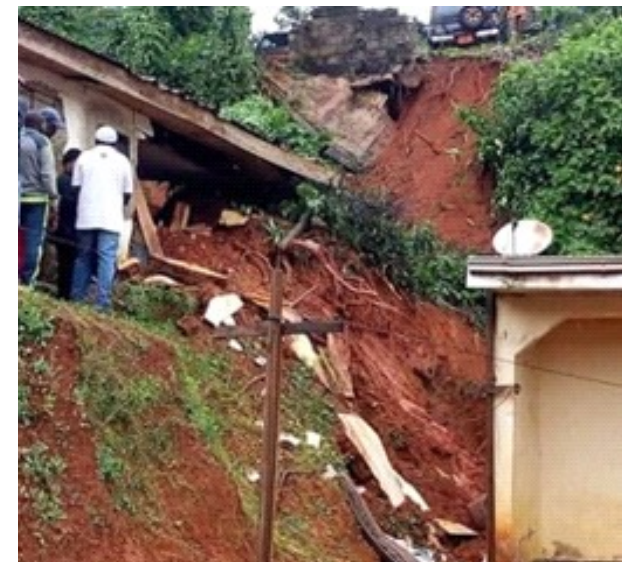

(2) Ngousso à Yaoundé en novembre 2019

Figure 8 : Illustrations d'éboulements au Cameroun

\subsection{La déprise accentuée des espaces}

\section{périphériques des villes}

La construction est le premier acte de déforestation et de déboisement, qui remplace le couvert végétal par des infrastructures imperméabilisant le sol. Ceci est accentuée par l'étalement horizontale des villes vers leurs périphéries à cause de l'accroissement de la démographie urbaine.

D'après une analyse du FAO (Organisation des Nations Unies pour l'alimentation et l'agriculture) entre 1990 et 2000, la couverture végétale au Cameroun a diminué de $0,9 \%$ notamment autour de l'aire métropolitaine de ses grandes villes (V. Djoufack, 2011). Cette régression de l'espace rural entourant les villes s'explique par l'étalement horizontal des villes et l'exode rural. Pourtant, ces surfaces végétales sont très importantes en ce sens qu'elles participent significativement à la réduction de la chaleur urbaine (d'au moins $2^{\circ} \mathrm{C}$ selon sa densité) et entre autres permet d'approvisionner la ville par des circuits courts grâce à l'agriculture de proximité.

\section{Discussion}

Le changement climatique au Cameroun se mesure par la variation des paramètres climatiques dont les plus significatifs sont la température et l'hygrométrie dont les contributions à la survenue d'inondations et par ricochet sur la dégradation des infrastructures urbaines est sans équivoque. On note que la hausse des températures entraine d'après les spécialistes l'augmentation de l'évapotranspiration contribuant à l'apparition des pluies plus longues et plus violentes. Les submersions qui en découlent dans les villes à relief accidenté, favorisent les glissements de terrain. On compte aussi la dégradation des routes et les déversements des égouts sur la voirie comme conséquences des inondations. Ces dernières attaquent également les fondations des bâtiments par l'action combinée de l'érosion et des poussées hydrauliques. L'humidification des murs des 
bâtiments et le décapage rapide des peintures s'en suivent rapidement. D'un autre côté, on a relevé que l'humidité de l'air ambiant a tendance à beaucoup varier concomitamment aux variations de températures et aux anomalies de la pluviométrie. Ce phénomène est d'autant plus perceptible que la ville est industrialisée et dépossédée de son couvert végétal jusqu’à sa périphérie. Les activités socio-économiques s'en trouvent fortement perturbées. Il faut noter que les groupes sociaux à faible revenu accroissent drastiquement la vulnérabilité de la ville car ils sont plus susceptibles de subir des conséquences mortelles en raison de leur mauvaise qualité de logement, faible accès aux soins et leur limite d'action en cas urgences (GIEC, 2012 ; M. Lawhon, 2014).

(GIEC, 2014b) nous fait remarquer que les Gouvernements n'auront jamais à eux seuls les ressources suffisantes pour empêcher les ravages du changement climatique. Compte tenu de la complexité des interactions entre l'urbanisation et l'évolution du climat urbain, ces derniers ne peuvent construire des stratégies efficaces qu'à travers une approche interdisciplinaire concertée de la communauté scientifique nationale encore aujourd'hui faiblement impliquée dans la recherche des solutions. A cela, il faudrait ajouter que les opérateurs économiques et les concessionnaires de réseaux techniques urbains ainsi que les populations ne sont pas sujets à suffisamment d'encadrements légaux en termes d'obligations ou de protections. Pourtant ce sont ces derniers qui doivent s'adapter aux énormes pertes de ressources qu'ils subissent afin de garantir un niveau de fonctionnement minimal des systèmes urbains en cas de catastrophes. Désormais, les axes stratégiques interpellant les secteurs impliqués par le développement urbain et la gestion du changement climatique doivent promouvoir la résilience urbaine.

\section{Conclusion}

Depuis le milieu du XXe siècle, les données climatiques relevées indiquent que l'atmosphère se réchauffe de plus en plus (GIEC, 2014a) et le niveau de la mer va sans cesse croissant. Ces variations de l'environnement se manifestent au Cameroun par l'augmentation des extrêmes de hautes températures, les perturbations des régimes pluvieux et la récurrence des vents violents amplifiés par la géographie contraignante de certaines villes et par l'influence anthropique sur l'environnement. Cette dernière s'illustre par la croissance urbaine rapide et la prolifération des bidonvilles qui couvrent désormais près de 65 à $70 \%$ de la superficie urbaine (LGSC, 2019). L'imperméabilisation accélérée du sol qui en découle couplée à la gestion insuffisante des déchets mettent à mal les ouvrages et infrastructures d'assainissement urbaines lorsqu'elles existent. Les conséquences se font davantage ressentir dans nos villes par les catastrophes, dont les multiples inondations enregistrées à ce jour n'en démontrent que le commencement. L'urgence de trouver des solutions durables à ce phénomène est désormais sans équivoque, et nécessite une encadrement cadre normatif inclusif plus performant protégeant équitablement les citadins nonobstant leur revenu. Ce qui passerait par un aménagement urbain et une production d'habitat respectueux des normes de sécurité et de santé (M. Lawhon, 2014).

\section{Remerciement}

Nos premiers remerciements vont à l'endroit de l'Académie des Sciences du Cameroun (ASC), pour avoir organisé la Conférence du 17 septembre 2020 à Yaoundé intitulée "Conférence du CAS à propos des catastrophes dans les villes dues au changement climatique », où nous avons traité du thème « changement climatique et urbanisation ». C'est à partir de cette conférence que nous avons saisi l'opportunité de mettre sur pieds cet article. 
Nous remercions ensuite le Laboratoire de Génie Civil et des Sciences de la Conception (LGSC) de l'ENSPY dirigé par Pr. Chrispin PETTANG, pour avoir nourri ces travaux de sa collection de mémoires de fin d'études de Master II de 1995 à 2019.

\section{Références}

[1] A. Amanejieu (2019). Analyse temporelle de la représentation du Risque d'inondation de 1980 à 2018 à Douala-Cameroun. Mémoire de Master de spécialisation en gestion des risques et des catastrophes. Faculté des Sciences, Université de Liège, $77 \mathrm{p}$.

[2] A. MAILHOT, S. DUCHESNE, J-P. VILLENEUVE (2007), Les changements climatiques : enjeux et perspectives en matière d'infrastructures urbaine, IRNS - Eau, Terre et Environnement, Québec, N62, 7p.

[3] A. Matcheubou, Elambo Nkeng, G., Tsalefac, M. and T. Tamo Tatietse (2009). "Problématique de la gestion urbaine et de la pollution de l'air en milieu urbain : le cas de Yaoundé." African Journal of Science and Technology 10(1): 1-15.

[4] A. Sharifi (2021). Co-benefits and synergies between urban climate change mitigation and adaptation measures: A literature review, Science of The Total Environment, Volume 750, 141642, ISSN 0048-9697, https:// doi.org/10.1016/j.scitotenv.2020.141642.

[5] BM (2017). Changement climatique et gestion des risques de catastrophe au Cameroun. Rapport diagnostic de la Banque Mondiale, juin 2017.

[6] B. Swynghedauw, J.-L. Weméau (2021). Rapport 20-07. Conséquences du changement climatique sur la santé humaine et animale, Bulletin de l'Académie Nationale de Médecine,
Volume 205, Issue 3, Pages 219-226, ISSN 0001 -

4079, https://doi.org/10.1016/ j.banm.2021.01.009.

[7] C. Brand et al. (2021). The climate change mitigation impacts of active travel: Evidence from a longitudinal panel study in seven European cities, Global Environmental Change, Volume 67, 102224, ISSN 0959-3780, https://doi.org/ 10.1016/j.gloenvcha.2021.102224

[8] C. Pettang (2001), Résorption de l'habitat spontané en milieu urbain : une approche basée sur des outils d'aide à la décision, Mémoire d'habilitation à Diriger les recherches; INSA de Lyon et Université Claude Bernard LYON 1121 pages.

[9] C. Pettang, (1998). Diagnostic de l'habitat urbain au Cameroun (1998) Urbanisme et Habitat volume 1 ; Presses Universitaires de Yaoundé 180 Pages

[10] CUD - Groupe Huit - AS Consultants (2011), PDU DOUALA 2025

[11] CUY-AUGEA INTERNATIONAL IRIS CONSEIL - ARCAUPLAN (2008), PDU YAOUNDE 2020

[12] C. Wache (2018), Modélisation des ruissellements pluvieux à Yaounde : Cas des inondations de l'avenue Kennedy. Mémoire d'Ingénieur de conception de génie civil, Ecole Nationale Ecole Nationale Supérieure Polytechnique de Yaoundé, 101p

[13] D. Crawley (2008), Estimating the impacts of climate change and urbanization on building performance. US Department of Energy, Washington, DC, USA. ISSN 1940-1493 print/ ISSN 1940-1507 online 2008 International Building Performance Simulation Association 
(IBPSA) DOI: 10.1080/19401490802182079 http://www.informaworld.com

[14] E. Ngninkam et E. Tanawa (2006), Les villes d'Afrique face à leurs déchets, Université de Belfort-Montbéliard (UTBM), 281p.

[15] FICR -Fédération Internationale des Sociétés de la Croix-Rouge et du Croissant-Rouge (2010). Rapport Annuel 2010. Genève, Suisse. Disponible en ligne sur http://www.ifrc.org/ Global/Publications/annual-reports/IFRCAnnual-report-2010-French.pdf (consulté le 10/ 09/2020)

[16] G. TJOMB (2018). Stratégie de production de logements sociaux basée sur la réhabilitation des QHS. Mémoire d'ingénieur de conception de génie civil, École Nationale Supérieure Polytechnique de Yaoundé, 111p. [17] GIEC (2012). Special Report on Managing the Risks of Extreme Events and Disasters to Advance Climate Change Adaptation. A Special Report of Working Groups I and II of the Intergovernmental Panel on Climate Change. Cambridge University Press, Cambridge, UK and New York, NY, USA, 582 pp.

[18] GIEC (2020). Rapport spécial du GIEC sur le changement climatique et les terres émergées | Quels impacts pour l'Afrique ? 40p.

[19] GIEC, (2014) a. Climate Change 2014: Impacts, Adaptation, and Vulnerability. Part A: Global and Sectoral Aspects. Contribution du groupe de travail II au cinquième rapport d'évaluation du Groupe d'experts intergouvernemental sur l'évolution du climat. Cambridge/New York, Royaume-Uni/USA, Cambridge University Press. www.ipcc.ch/site/ as sets / uploads/2018/02/W GIIAR 5 PartA_FINAL.pdf
[20] GIEC, (2014) b. Climate Change 2014: Impacts, Adaptation, and Vulnerability. Part B: Regional Aspects. Contribution du groupe de travail II au cinquième rapport d'évaluation du Groupe d'experts intergouvernemental sur l'évolution du climat. Cambridge/New York, Royaume-Uni/USA, Cambridge University Press. www.ipcc.ch/site/assets/uploads/2018/02/ WGIIAR5-PartB_FINAL.pdf

[21] G. L'HOSTIS, (1987). Variabilité des précipitations dans la région du Mont-Cameroun et mécanisme de la mousson. Mémoire de DEA national d'hydrologie - Filière "Ressources en eau". Laboratoire de Géochimie Isotopique, Université d'ORSAY.

[22] Hallegatte, Stéphane \& Green, Colin \& Nicholls, Robert \& Corfee-Morlot, Jan. (2013). Future flood losses in major coastal cities. Nature Climate Change. 3. 802-806. 10.1038/ nclimate1979.

[23] H. Waisman (2020). Quelles transitions pour l'atténuation du changement climatique ? Transformations globales, enjeux sociétaux, et leçons pour la decision. Comptes Rendus. Géoscience, Tome 352 (2020) no. 4-5, pp. 319328

[24] INS (2015). Annuaire Statistique du Cameroun 2015, Cameroun.

[25] J. L. Nsouandélé, D. K. Kidmo, S. M. Djetouda and N. Djongyang (2016). Estimation statistique des données du vent à partir de la distribution de Weibull en vue d'une prédiction de la production de l'énergie électrique d'origine éolienne sur le Mont Tinguelin à Garoua dans le Nord Cameroun. Revue des Energies Renouvelables Vol. 19, N² (2016) pp.291 - 301. 
[26] J. Nyaka (2017). Elaboration d'un Modèle Adapté aux Contraintes du Développement Durable en vue du Dimensionnement des Sections Hydrauliques des Canaux d'Assainissements des Eaux Pluviales. Mémoire d'ingénieur de conception de génie civil, Ecole Nationale Supérieure Polytechnique de Yaoundé, $62 \mathrm{p}$.

[27] L. Bruckmann, A. Amanejieu, M. Zogning Moffo et P. Ozer (2020), « Analyse géo historique de l'évolution spatio-temporelle du risque d'inondation et de sa gestion dans la zone urbaine de Douala (Cameroun)», Physio-Géo [En ligne], Volume 13 | 2019, mis en ligne le 04 septembre 2019, consulté le 05 septembre 2020. URL: http://journals.openedition.org/physiogeo/8038; DOI: https://doi.org/10.4000/ physio-geo. 8038

[28] L. Cui et J. Shi (2012), Urbanization and its environmental effects in Shanghai, China. Elsevier, Urban Climate. http://dx.doi.org/ 10.1016/j.uclim.2012.10.008

[29] LGSC (2019). Collections des Archives du Laboratoire de Génie Civil et des Sciences de la conception, de 1995 à 2019. Ecole Nationale Ecole Nationale Supérieure Polytechnique de Yaoundé.

[30] M. Lawhon, (2014). Diana Mitlin and David Satterthwaite 2013: Urban Poverty in the Global South: Scale and Nature. London and New York: Routledge. International Journal of Urban and Regional Research. 38. 10.1111/14682427.12137_6

[31] MINEPDED (2015), Plan National d'Adaptation aux Changements Climatiques (PNACC). République du Cameroun.
[32] Munich Re Group (2004); Annual Report 2004. Münchener RückversicherungsGesellschaft Königinstrasse 107. 80802 München Germany. Disponible sur http:// www.munichre.com

[33] O. Leumbe et al. (2015). Cartographie des zones à risques d'inondation en zone soudanosahélienne : cas de Maga et ses environs dans la région de l'extrême-nord Cameroun. A frique SCIENCE 11(3) pp.45-61. ISSN 1813-548X.

[34] ONU (2016), Rapport sur les Objectifs du Développement Durable 2016.

[35] ONU-HABITAT (2007), PROFIL URBAIN NATIONAL DU CAMEROUN 20062007, 28p.

[36] ONU-HABITAT (2014), THE STATE OF AFRICAN CITIES 2014: Re-imagining sustainable urban transitions, 278p.

[37] P. Tchawa (2012), Le Cameroun : une « Afrique en miniature »?, Les Cahiers d'Outre-Mer [En ligne], 259 | Juillet-Septembre 2012, mis en ligne le 01 juillet 2015, consulté le 05 septembre 2020. URL : http://journals.openedition.org/ com/6640 ; DOI : https://doi.org/10.4000/ com.6640

[38] PNUD (2008). Rapport Annuel 2008, www.cm.undp.org; ONU

[39] Ren Guo-Yu (2015). Urbanization as a major driver of urban climate change, Editorial / Advances in Climate Change Research 6, http:// dx.doi.org/10.1016/j.accre.2015.08.003.

[40] S. Andrei (1977). Propriétés hydrauliques et mécaniques des sols non saturés. Laboratoire de Mécanique des Sols, Université de Constantine et Institut de Construction, Bucarest, Roumanie. 
[41] S. Balica $\bullet$ N. Wright $\bullet$ F. van der Meulen (2012). A flood vulnerability index for coastal cities and its use in assessing climate change impacts. Springer. Nat Hazards (2012) 64:73-105. DOI 10.1007/s11069-012-0234-1. Disponible sur https://link.springer.com/content/pdf/ 10.1007/s11069-012-0234-1.pdf (consulté le $15 / 09 / 2020)$

[42] S. Kuetche Fotsi (2013). Récurrence des inondations a Yaounde et influence des facteurs physiques ou anthropiques : cas du bassin versant de la BIYEME (Cameroun). Exposé au 18e colloque international en évaluation environnementale.

[43] UNISDR (2009). UNISDR Terminology on Disaster Risk Reduction. United Nations. International Strategy for Disaster Reduction (UNISDR), United Nations, Geneva, Switzerland, $30 \mathrm{pp}$.

[44] V. Djoufack (2011). Étude multi-échelles des précipitations et du couvert végétal au Cameroun : Analyses spatiales, tendances temporelles, facteurs climatiques et anthropiques de variabilité du NDVI. Océan, Atmosphère. Université de Bourgogne.

[45] V. LEFEBVE (2020). "Dérèglement climatique et coronavirus, d'une urgence à l'autre ", Les@ @nalyses du CRISP en ligne, consulté le 02 sept.-2021, www.crisp.be

[46] Https://www.populationstat.com/ cameroon/ consulté le 06/09/2020.

[47] Https://www.editions2015.com/ cameroun/cartes.php consulté le 16/09/2021

\section{Liste des abréviation}

ASC Académie des Sciences du Cameroun CCHST Centre Canadien d'Hygiène et de Sécurité au Travail

FAO Organisation des Nations Unies pour l'alimentation et l'agriculture

FICR Fédération internationale des sociétés de la Croix-Rouge et du Croissant-Rouge

GIEC Groupe d'experts Intergouvernemental sur l'Evolution du Climat

INS Institut National de la Statistique

LGSC Laboratoire de génie Civil et Sciences de la Conception

MINEPDED Ministère de l'Environnement, de la Protection de la Nature et du Développement Durable

ONU Organisation des Nations Unies

PED Pays en voie de développement

PNACC Plan National d'Adaptation aux Changements Climatiques

PNUD Programme des Nations Unies pour le Développement

QHS Quartier à Habitats Spontanés

UNISDR United Nations. International Strategy for Disaster Reduction 